\title{
Convolutional Neural Network Integrated With Fuzzy Rules for Decision Making in Brain Tumor Diagnosis
}

\author{
Pham Van Hai, Hanoi University of Science and Technology, Hanoi, Vietnam \\ Samson Eloanyi Amaechi, Hanoi University of Science and Technology, Hanoi, Vietnam
}

\begin{abstract}
Conventional methods used in brain tumor detection, diagnosis, and classification such as magnetic resonance imaging and computed tomography scanning technologies are unbridged in their results. This paper presents a proposed model combination, convolutional neural networks with fuzzy rules in the detection and classification of medical imaging such as healthy brain cells and tumor brain cells. This model contributes fully on the automatic classification and detection of medical imaging such as brain tumors, heart disease, breast cancer, HIV, and flu. The experimental result of the proposed model shows overall accuracy of $97.6 \%$, which indicates that the proposed method achieves improved performance over the other current methods in the literature such as classification of tumors in human brain MRI using wavelet and support vector machine (94.7\%) and deep convolutional neural networks with transfer learning for automated brain image classification (95.0\%) used in the detection, diagnosis, and classification of medical imaging.
\end{abstract}

\section{KEYWORDS}

Brain Tumors, Convolutional Neural Networks, Fuzzy Rule, Image Classification, Medical Diagnostics

\section{INTRODUCTION}

The current World Health Organization (WHO) guidelines for brain tumor classification are strictly histopathological, which limits clinical application (Geethu Mohan et al.,2018). This has a constraint in the field of medical imaging for diagnosis and classification planning including automated approaches. Approaches have been abandoned in favor of non-invasive, high-resolution techniques, especially magnetic resonance imaging (MRI) and computed tomography (CT) scans (Thomas et al., 2018), though MRI is typically the reference standard used (Michael et al.,2018). Analyses of large-scale medical imaging data involving deep learning are rapidly evolving to include classification, detection, and diagnosis. Diffusion tensor imaging (DTI) is a non-invasive method that includes the use of MRI, allowing the study of white matter and therefore of the corticospinal tracts (Domenico Chirchiglia et a.,2020). Currently, considerable attention has been paid to magnetic particle imaging (MPI)because of its better sensitivity compared to MR. Imaging technologies have played a role in the analysis of brain anatomy and functions with the development of technology (LakshmanaprabuS.K.,2019). Early imaging brain tumor treatment approaches, such as pneumonia cephalography and cerebral angiography are invasive and potentially dangerous. 
Recently, an important diagnostic technique in medical imaging is a non-invasive method uses by clinicians to diagnose and identify the development of brain tumors. Brain tumors can be examined using computed tomography (CT) or magnetic resonance imaging (MRI), the results are often confirmed with a biopsy an invasive procedure). Computer tomography (CT) is an imaging procedure that creates detailed images of scans of areas inside the body. It is often termed computerized tomography or computerized axial tomography (CAT). MRI is an imaging technology producing threedimensional detailed anatomical images, it is used in both diagnosis and treatment monitoring. MRI is based on sophisticated technology that excites and detects the change in the direction of the rotational axis of protons found in the water that makes up living tissues. The theory of image segmentation and extraction in brain tumors plays a vital lot in the diagnosis of tumors and the application has created a big gap in the monitoring of tumor grades between computers and clinicians. Furthermore, radiologists improve diagnostic accuracy from a different perspective in interpreting medical images with these hybrid techniques (Mohan $\mathrm{G}$ et al,2018). The use of a convolutional neural network (CNN) technique in the analysis of automatic medical images has gained significant adhesion as an effective approach in medical diagnostics. The processing of medical images currently plays a central role in the diagnosis and monitoring of a broad range of medical conditions and has the benefit of identifying such conditions at an early stage to enable appropriate treatment (P.Mohamed Shakeel et al, 2019). Image classification, extraction, and segmentation are in all areas of medical examination to solve complicated problems in medical images in hospitals (Tuan, et al, 2017). The literature has a large body of published research addressing the classification of medical images including significant reported results relating to the classification of brain tumors. However, the classification methods have not achieved $100 \%$ accuracy in the areas of medical imaging classification.

This paper suggests a method to hybrid a convolutional neural network with a fuzzy rule in medical image analysis and classification of brain tumors based on normal brain cells or abnormal brain cells. The model was applied in the detection and classification of the presence of brain cells and the accuracy of the results. Using a convolutional neural network combined with fuzzy rules in medical images helps in increases of tumor diagnosis and classifying the basement of the tumor. In this paper, a comprehensive feature set and fuzzy rules are selected to classify an abnormal image to the corresponding tumor type. The experimental results show the proposed (CNN- FL) model of imaging detection and classification of brain tumors based on the normal and abnormal brain cells.

The structure of the paper is as follows: section 2 presents related research, section 3 presents materials and methods, section 4 presents the proposed model, section 5 presents experimental results and discussion, and section 6 presents the conclusion and future work.

\section{RELATED RESEARCH}

Application of convolutional neural network combined with fuzzy rules has significant potential impacts in classification and detection of medical imagines. Badran et al (Ehab F. Badran et al.,2010) have proposed a computer-based method using an algorithm to analyze MRI images to detect abnormal brain cells. The method focuses on how to classify the brain cells into healthy brain cells and affected brain cells including classifying tumors as benign or malignant. The model identified the uses of image enhancement and segmentation in the classification of brain tumors through neural networks and the backpropagation network method. The theory proves that the brain tumors in the MRI image of patients can be detected using this proposed model.

Bhanumurthy et al (M.Y.Bhanumurthy et al.,2015), have proposed an automated technique using artificial intelligence to detect and segment abnormal tissues using tumors and atrophy in brain MRI images. The model application proves that energy, entropy, homogeneity, and correlation, work together in brain MRI images to classify healthy (normal) brain cells and abnormal(tumor) brain cells. The method uses a support vector machine (SVM) in a Neuro-fuzzy classifier which is claimed to be faster and is computationally efficient than alternative methods. The model showcases a good accuracy of 
94.7\%.In a study by Yang et al (Aimin Yang et al.,2019), an automatic brain tumor detection model using a convolutional neural network (CNN) algorithm for classification of brain images based on tumor and non-tumor detection. The model achieved $79.5 \%$ accuracy using a convolutional neural network $(\mathrm{CNN})$. This method is compared with other alternatives methods in the classification of brain tumors such as fellows: classification of tumors in human brain MRI using wavelet and support vector machine $94.7 \%$ with error rate $5.3 \%$, and deep convolutional neural networks with transfer learning for automated brain image classification $95.0 \%$ with error rate $5.0 \%$, use in the detection, diagnosis, and classification.

In (2019) Sugimori et al (Hiroyuki Sugimori et al.,2019) have proposed a model of convolutional neural network (CNN) in the detection of brain tumors. These investigations use a method of the standard line for brain magnetic resonance imaging, the goal of the research is to create a detector able to identify the anatomical structure of the brain using an MRI scan to draw a standard line. In considering the proposed model with other alternative related publications point out the advantages and disadvantages of the current proposed model. Moreover, the comparing factors are based on fellows: dataset, accuracy, algorithms, and problems. All these factors are shown in the table 1, the analysis focuses on the current publication in the year 2020 based on brain tumor classification, detection, and optimization. Based on the analysis the researcher can focus on its results as proof in any future research work.

\section{MATERIALS AND METHODS}

\subsection{Dataset}

MRI (Magnetic Resonance Imaging) is a scanner that uses powerful magnets to polarize excite hydrogen nuclei (a single proton) in human tissues, produces a single proton that can be detected and encoded spatially in an image of the human body. In the model, our experiment uses a dataset collected from the Web site [http://www.diacom.com]. The dataset consists of a total of $3264 \mathrm{~T} 1-$ CE MRI images with three different brain tumor types: 100 brain tumor images of glioma tumor, 115 brain tumors of meningioma tumor,74 brain tumors of pituitary tumor, and 105 brain cells without tumor. These images are divided into three categories, $70 \%$ for training, $15 \%$ for testing, and $15 \%$ for validation. The experiment was categorized into different stages in other to enhance and improved the experimental results.

\subsection{The Fuzzy Rules Concept}

Definition: Fuzzy rule sets are represented as shown in eq. (1):

$\mathrm{A}=\left\{\left(\mathrm{x}, \mu_{\mathrm{A}}(\mathrm{x})\right) \mid \mathrm{x} \hat{\mathrm{I}} \mathrm{X}\right\}$

where $\mu \mathrm{A}[1,0]$ satisfy the condition as shown in eq. (2):

$\mathrm{A}=\{\langle\mathrm{x}, \mu \mathrm{A}(\mathrm{x})\rangle \mid \mathrm{x} \hat{\mathrm{I}} \mathrm{X}\}$

where $\mu \mathrm{A}: \mathrm{X} \AA[0,1]$ is the membership function of the FS

$\mathrm{A}, \mu \mathrm{A}(\mathrm{x}) \hat{\mathrm{I}}[0,1]$ is the degree of membership of the element $\mathrm{x}$ to the set $\mathrm{A}$.

A fuzzy number denoted as $U=\{\langle x, \mu A(x), \nu A(x)\rangle \mid x \hat{I} X$ IR $\}$ is shown in equation [2] is the membership function of the input variable for the classification of brain cells (and brain tumors).For a set $\mathrm{X}$ an input variable as it relates to Atanassov is given by eq. (3):

$\mathrm{A}=\{\langle\mathrm{x}, \mu \mathrm{A}(\mathrm{x}), \nu \mathrm{A}(\mathrm{x})\rangle \mid \mathrm{x} \hat{\mathrm{I}} \mathrm{X}$ IR $\}$ 
Table 1. Evaluation and comparison of related publication (2020)

\begin{tabular}{|c|c|c|c|c|c|}
\hline Paper & Tasks & Architecture & Dataset & Accuracy & Problems \\
\hline $\begin{array}{l}\text { 1.RahelehHashemzehia et } \\
\text { al, } 2020\end{array}$ & $\begin{array}{l}\text { Classification, } \\
\text { identification }\end{array}$ & $\begin{array}{l}\mathrm{CNN} \text { and } \\
\mathrm{NADE}\end{array}$ & CE-MRI & $95.0 \%$ & $\begin{array}{l}\text { A high classification } \\
\text { performance as } \\
\text { regards the availability } \\
\text { of medical images are } \\
\text { limited of the brain } \\
\text { tumors }\end{array}$ \\
\hline 2. T. Maekawa et al.,2020 & Classification & $\begin{array}{l}\text { OGSE and } \\
\text { PGSE }\end{array}$ & MRI dataset & $97.0 \%$ & $\begin{array}{l}\text { To distinguishing low- } \\
\text { grade and highgrade } \\
\text { brain tumors. }\end{array}$ \\
\hline $\begin{array}{l}\text { 3. Ahmet Çinar et al., } \\
2020\end{array}$ & Detection & $\begin{array}{l}\text { CNN and } \\
\text { Resnet50 }\end{array}$ & $\begin{array}{l}\text { Brain MRI } \\
\text { Images from the } \\
\text { Kaggle Site }\end{array}$ & $97.2 \%$ & $\begin{array}{l}\text { An effective and can } \\
\text { be used in computer- } \\
\text { aided systems to } \\
\text { detect brain tumor }\end{array}$ \\
\hline $\begin{array}{l}\text { 4.PRIYANSH SAXENA } \\
\text { et al., } 2020\end{array}$ & Classification & $\begin{array}{l}\mathrm{CNN} \text {, and } \\
\text { transfer } \\
\text { learning }\end{array}$ & $\begin{array}{l}\text { MSG/SEVIRI } \\
\text { satellites }\end{array}$ & $90.0 \%$ & $\begin{array}{l}\text { An approach to } \\
\text { classifying brain } \\
\text { tumor MRI images } \\
\text { into cancerous or non- } \\
\text { cancerous by utilizing } \\
\text { the concepts of image } \\
\text { processing, }\end{array}$ \\
\hline $\begin{array}{l}\text { 5. Sharan Kumar et al., } \\
2020\end{array}$ & $\begin{array}{l}\text { Optimization/ } \\
\text { Classification }\end{array}$ & $\begin{array}{l}\text { Dolphin-SCA } \\
\text { and Deep } \\
\text { CNN }\end{array}$ & BRATS dataset & $96.3 \%$ & $\begin{array}{l}\text { To improve the } \\
\text { accuracy and to make } \\
\text { effective decisions in } \\
\text { classification. }\end{array}$ \\
\hline 6.Fatih Özyurt et al,2020 & Detection & $\begin{array}{l}\text { CNN and SR- } \\
\text { FCM }\end{array}$ & $\begin{array}{l}\text { Cancer } \\
\text { Genome Atlas } \\
\text { Glioblastoma } \\
\text { Multiform } \\
\text { (TCGA-GBM) }\end{array}$ & $98.33 \%$ & $\begin{array}{l}\text { To segmented the } \\
\text { tumors in high } \\
\text { performance. }\end{array}$ \\
\hline $\begin{array}{l}\text { 7.NavidGhassemi Fong et } \\
\text { al,2020 }\end{array}$ & Classification & $\begin{array}{l}\text { CNN and } \\
\text { GAN }\end{array}$ & MRI dataset & $95.6 \%$ & $\begin{array}{l}\text { To increased the } \\
\text { overall performance } \\
\text { intensely. }\end{array}$ \\
\hline
\end{tabular}

where the functions $\mu \mathrm{A}: \mathrm{X}{ }^{\circledR}[0,1]$ and $\nu \mathrm{A}: \mathrm{X} \AA[0,1]$, with the condition $0 £ \mu \mathrm{A}(\mathrm{x})+\nu \mathrm{A}(\mathrm{x}) £$ $1, \forall \mathrm{x} \hat{\mathrm{I}} \mathrm{X}$.The numbers, $\mu \mathrm{A}(\mathrm{x}) \hat{\mathrm{I}}[0,1]$ and $\nu \mathrm{A}(\mathrm{x}) \hat{\mathrm{I}}[0,1]$ denotes the degree of membership and the degree of non-membership of the element $\mathrm{x}$ to the set a respectively.

\subsection{Convolutional Neural Networks Approach}

A convolutional neural network (CNN) is one of the classes of deep feed-forward artificial neural networks $(\mathrm{NN})$ that employs a multilayer perceptron structure. It was invented and motivated by biological processes with connectivity patterns between neurons and also inspired by the organization of the animal visual cortex. A convolutional neural network (CNN) consists of a three-layer, a convolutional layer, a pooled layer, and a fully connected layer. They have applications in image and video recognition, recommender systems, image classification, medical image analysis, and natural language processing (Collobert et al.,2018). Convolutional neural network (CNN)uses pre-processing image classification and make a comparison with other algorithms; this means that the networks learn the filters that in traditional algorithms hand made. CNN's are more difficult to build than classical neural networks, however, they are easier to use (Saroj Kumar Chandra et al.,2019). The structure in Figure 1, shown the diagram convolutional neural network (CNN). 

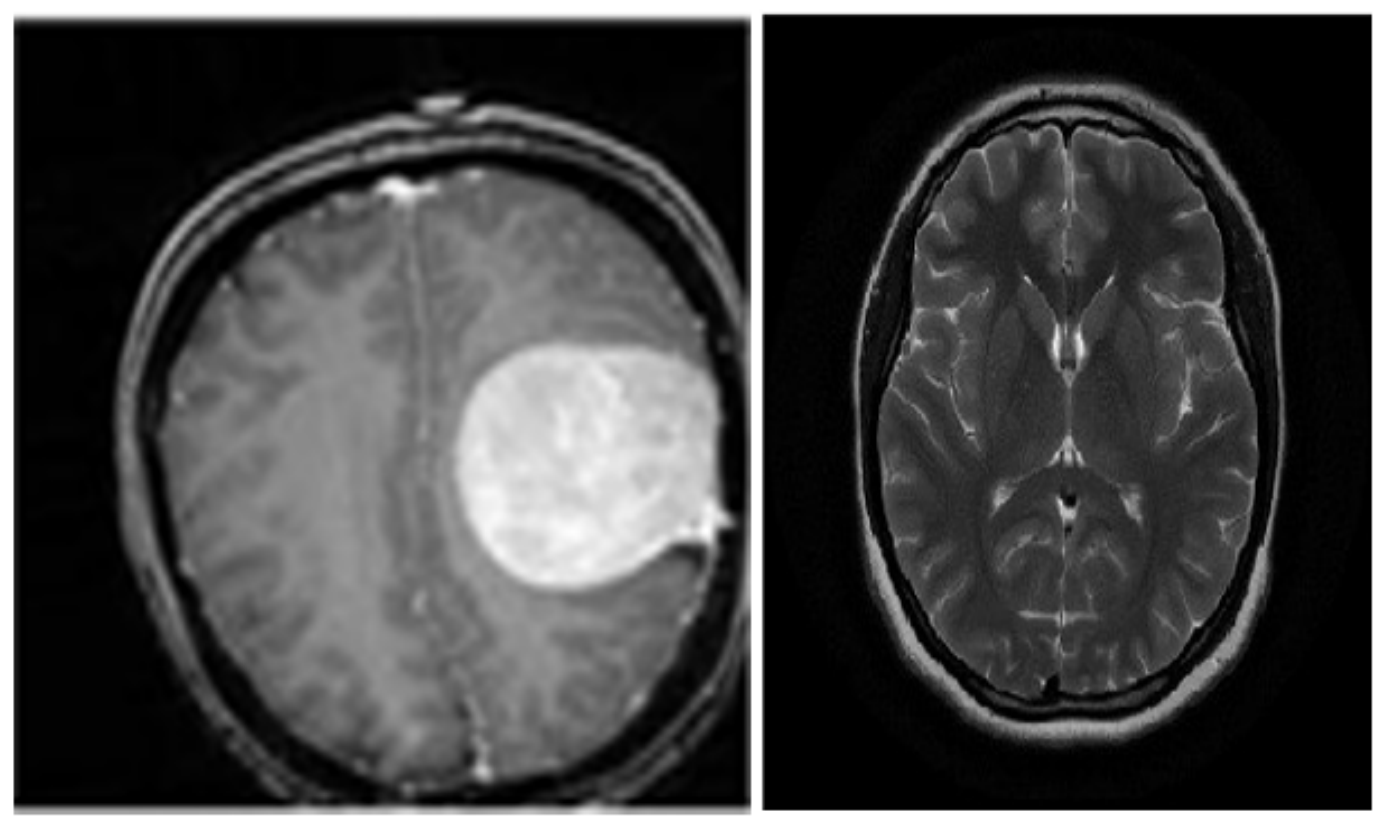

A CNN is a deep learning neural network technique that employs an arithmetical operation in its implementation as such convolution. They run with an activation function; the aim of the common simple activation function is a rectified linear unit (RELU) layer which is essential to the success of deep neural networks (DNNs) (Xiaoheng Jiang et al., 2018). The rectifiers are an activation function shown in eq. (3):

$\mathrm{f}(\mathrm{x})=\mathrm{x}+\max (0, \mathrm{x})$

where $\mathrm{x}$ is the input to a neuron.

The RELU layer is an element-wise process that operates on pixels with substitutes of all the negative values in their mapping system and feature map of zero. Presently, the RELU is the most powerful and popular activation function for the improvement of convergence from the hyperbolic tangent function. Such networks are commonly trained under a log loss (or cross-entropy) regime, giving a non-linear variant of multinomial logistic regression (Bishop, Christopher M, et al., 2006). The RELU layer is an activation function that consists of the following: pooling layers, fully connected layers, and normalization layers.

(A) Pooling Layer: It's used to reduce feature map dimensions of the data by connecting with outputs of neuron in the next layer. They provide an approach to downsampling feature maps and summarized the presence of patches in the area of the feature map.

(B) Fully connected Layers: They connected the neuron layer to the neuron layer in the cell. It has a traditional multi-layer perceptron that employs a softmax activation function in the output layer. The output leads by the convolutional and pooling layers create high-level features of the input data, which is used in the feature for classifying the input data into various classes in classification problems. A fully connected layer is just linked to a linear output (Krizhevsky A et al.,2012). 
(C) Dropout layer: It is the technique uses to improve out-fit on neural networks. It's applied to control overfitting on neural networks by restricting complex co-adaptation on training data. In the regularization technique, we use it both hidden and visible, randomly, by a specific know rate will be dropped out, during the training phase (Srivastava $\mathrm{N}$ et al., 2014). They have the effect of making the training data process noisy and the ability to force nodes within a layer to probabilistically take on more for the inputs.

\subsection{Fuzzy Logic (FL) and Fuzzy Rules}

Fuzzy logic is a mathematical model based on the fuzzy set theory and uses operational and control laws in its implementation. Its application involves reasoning approaches in a combination of medical imaging with unsharp, masking, and filter preprocessing. Its process results in reduced noises, sharpened edges of tumors, and the overall classification of brain cells based on health cells or tumors cell. The system helps to determine the medical imaging standard such as the condition of brain cells, whether a tumor using abnormality cluster or mass effect using cerebrospinal fluid. For approximate reasoning, the rule base considered three parameters namely patient age, tumor shape (mass or cystic), and mass effect existence (Geethu Mohan et al,2018).

Brain tissues are extraordinary and complex, the manual distinction of brain tumors from normal brain tissue is so hard for a medical professional but in this system, we build knowledge of using Adaptive Neuro-Fuzzy inference System(ANFIS) based on the automatic gray point selected range in brain tissue but in the referred work (Zheng Huang et al.,2020).To extract complete features of brain tissues such as edges and remove noise, the theory of fuzzy rule, degree of membership that is intermediary between 0 and 1 is applies.

To initiate fast convergent time, during the inference engine and described the nature of brain tissues in the rules extraction we have applied the fuzzy rule reasoning which based on fuzzy set theory (Moore et al, 2012). It's compared the initial brain tissues and the extracted brain tissues with CNN and fuzzy set theory (S. Roy et, al.,2016). Output section where the images are classifiers, $\mathrm{W}_{0}$ membership functions (high and low) were used in if-then rules forming the output.

The theory of fuzzy rules is implemented in the brain tumors: IF-THEN rules map input or computed truth values to desired output truth value as shown in Figure 2.

Figure 2. Fuzzy Rules Theory

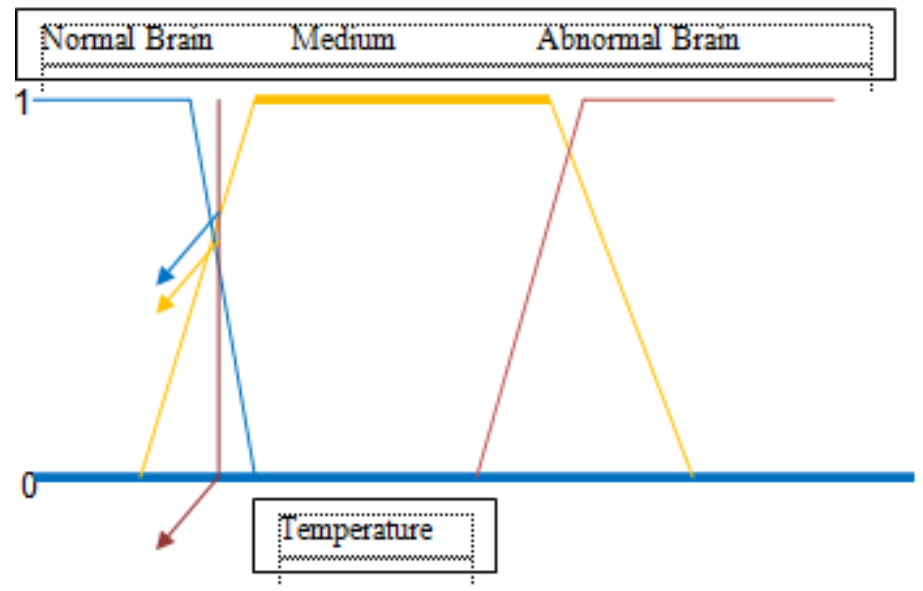




\section{IF - THEN RULES}

IF Brain is hot

THEN tumor present (Abnormal Brain)

IF Brain is low -------THEN absence of tumor (Normal Brain)

IF Brain is medium ----- THEN analyzed the condition of Brain whether (Abnormal or normal)

\section{THE PROPOSED MODEL}

In this paper, the proposed a model of convolutional neural network $(\mathrm{CNN})$ integrated with fuzzy rules (FL) in the classification for medical imaging such as brain tumors. The architecture diagram model is shown in Figure 3.

The proposed model (CNN-FL) consists of five sections such as fellows: input data, image preprocessing, image segmentation, feature extraction, and integration section of (CNN-FL). The model process is described as follows:

\section{Step1: Input Data}

MRI data image was collected from the Web site [http://www.diacom.com]. The dataset consists of a total of 3264 T1-CE MRI images with three different brain tumor types:100 brain tumor images

Figure 3. Proposed Model (CNN- FL)

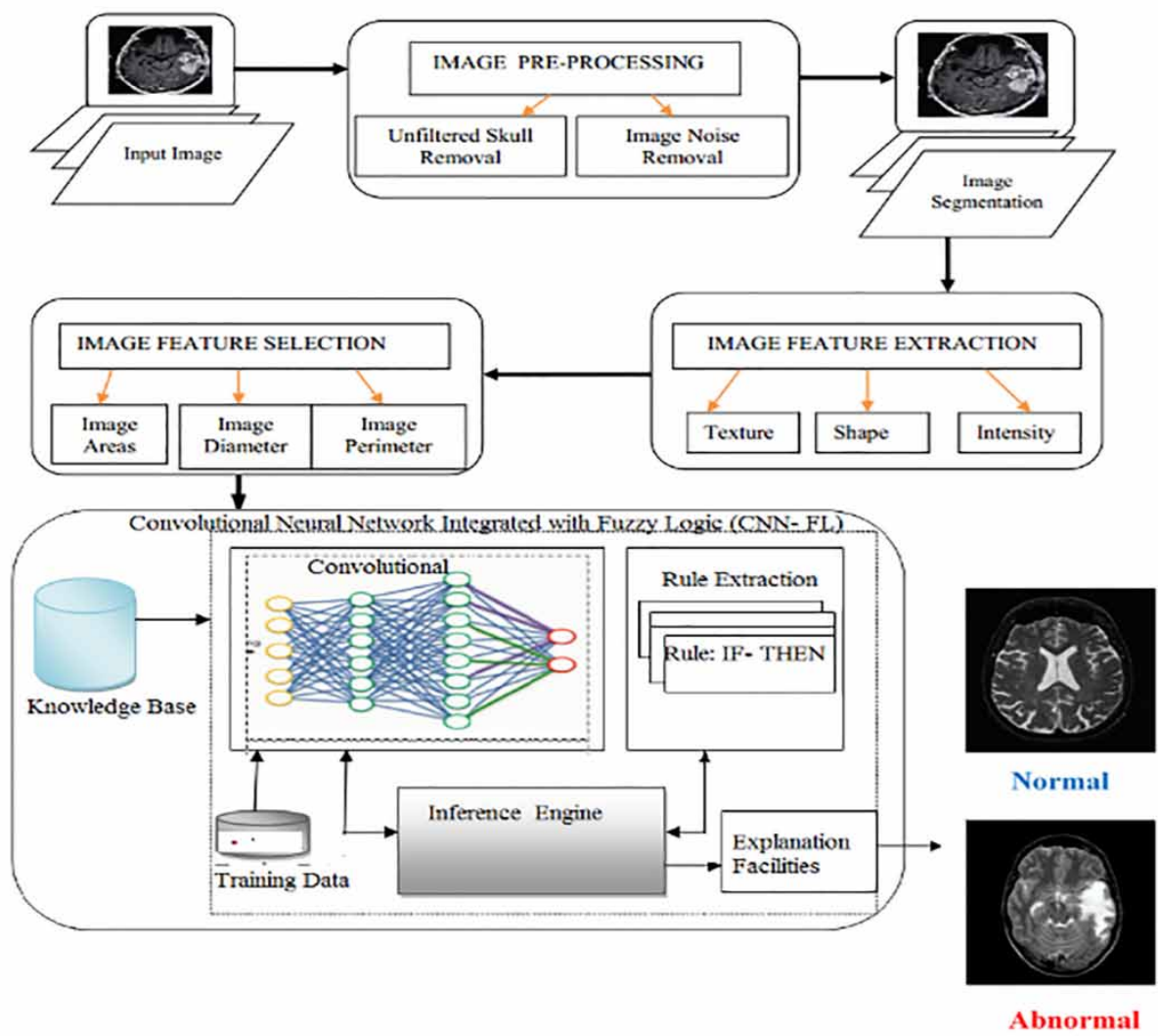

Output Classes 
of glioma tumor, 115 brain tumors of meningioma tumor,74 brain tumors of pituitary tumor, and 105 brain cells without tumor. These images are divided into three categories, $70 \%$ for training, $15 \%$ for testing, and $15 \%$ for validation. Each data image occupies a size of $256 \times 256$ pixels in the axial plane.

Step 2: Image Preprocessing

It requires the removal of unwanted features in the brain before diagnosis. Many times, bias is present in the scanning results, which affect the segmentation results, particularly in the computerbased models. On the other hand, it started by removing noise and rearranged the image quality, which involves using a filter process method. After filter, fellows by the masking skull of the brain to scrutinize the tumors. Mask sculling function in three ways:(1) To remove the known brain portion; (2). Improves the quality of tumor investigation regions. (3) It exposes the features of the brain for radiologists to view and identifying prominent structures of the brain. We also applied the threshold value process to get the desired quality of the brain.

Step 3: Image Segmentation

Segmentation is one of the most important steps in this current proposed model because it involves divides the MRIs into areas of interest. A threshold method of segmentation is applied in the segmentation stage with a combination of the binarization approach. After that, the input image was converted into its binarization format which completely changes to the binarization threshold. Image-based segmentation is gaining much interest in the diagnosis of infection (M. A. Khan et al.,2019). During the segmentation we have some challenges such as fellows: contrast lesions, change of tumor shape, change in texture exist which degrades the segmentation performance. Nevertheless, segmentation is used to separate multiple images from the object region especially in the brain tumor where the brain is joined with other cells. The threshold method is one of the best processes to separate the brain tumor from the cell. Automatic segmentation of brain tumors from medical images is important for clinical assessment and treatment planning of brain tumors (Alpana Jijja et al.,2017). The semi-automatic and fully automatic segmentation of tumor brain images are faced with great challenges due to usually exhibiting unclear and irregular boundaries with discontinuities and partial-volume effects for brain tumor images (Gursangeet Kaur et al., 2016).

Step 4: Image Extraction

Image features have been extracted by using a discrete Glimpse. Glimpse converts images to the frequency domain by filtering the image with a low pass and a high pass filter. Glimpse divides the image into approximate coefficients such as lower layers and higher layers and so. On the other hand, we pass brain tumors to feature extraction to identified brain tumor position and exact location when a diagnosis is processing. The elements of the feature vectors are the lengths of vectors that originate from the center of the mass and end up to the brain tumor tips. The geometrical feature of the brain tumor is given as follows:(1)Area of image,(2)Perimeter of image,(3)Centroid of the image,(4) Diameter of the image

In this practice do to the large volume of the dataset, feature extraction is the transformation of input data into a set of features, normally distinctive properties of input patterns that differentiate between many inputs of patterns (Y.Faridah et al.,2017).

Step 5: Image Feature Selection

It is necessary to select the features that needed to be extracted to minimize the computation and optimized the time required for extraction. Applying the knowledge of component analysis is a complete approach for transforming the input image feature and to avoid the curse of dimensionality. In the model, we use feature selection to map out the area of concentration of the tumor. Thirteen GLCM features, namely, contrast, correlation, energy, entropy, homogeneity, inverse difference moment (IDM), mean, variance, root mean square (RMS), standard deviation, smoothness, kurtosis, and skewness have been selected for the two classification stages for tumor detection and classification (Mahmoud Khaled et al.,2018).

Step 6: Convolutional Neural Network and Fuzzy Logic (CNN-FL) 
The integration of the convolutional neural network and fuzzy rules commenced immediately after the image feature selection of the image. The CNN-FL consists of the fellows, the knowledge base, convolutional, extraction rule, inference engine, and explanation facilities. According to Figure 3 , the knowledge base is a central repository for information on the dataset. All the input data was a store in the knowledge base for precisely matching the data during the training network. The system uses a necessary component in the training data, as the knowledge base is used to optimize information collected and process it for constant use in the convolutional stages. The convolutional stage comes immediately after the input data from the knowledge base is prepared to implement. It's composed of multiple layers that feature maps out input and provides a new feature mapping. The convolutional consists of three layers: a convolutional layer, a Relu layer, and a pooling layer. The convolutional layer was applied to map out the features of the brain tumors images and convolve the features to the next layer. The pooling layers reduce the multiple dimensions of the input image and modify the features for the next processing. The relu layers use the features created by the pooling layers and classify the images. Then, the extraction rule presents a conditional part to each rule with the given data in the database. Moreover, the condition of IF part of the rule matches the data in the database, the rule is eliminated and its THEN part is exected. This condition is continue exected until all the dataset in the database is completed executed which sets the condition of the data in the database. After, extraction rule the inference engine is set up to controls all information extracted from the system and initiates it with the rules base IF and THEN. This ensures complete reasoning in the system and creates a decision to ensure that brain tumors can implement according to the decision of fuzzy rules. Besides, the explanation facilities explain the condition and notify the outcome of classification based on the knowledge reasons for the inference engine. It creates the credibility of an expert system's conclusions is greatly enhanced by persuading the user that the information is correct. If the users can compare their reasoning with that of the expert system, and see that it agrees with their own, it is likely the advice will be accepted.

The proposed model CNN-FL utilizes the principle of the neuro-fuzzy system in its implementation as shown in Figure 4. A a neuro-fuzzy system is a neural network which implementations are equivalent to a fuzzy inference model. They trained to utilize (IF-THEN) rules and determine membership function for input and output variables of the system. Neuro-fuzzy is a neural complementary tool in building intelligent systems. While neural networks are low-level computational structures that perform well when dealing with raw data, fuzzy logic deals with reasoning on a higher level, using linguistic information acquired from domain experts. On the other hand, although neural networks can learn, they are opaque to the user. Besides, a convolutional neural network integrates with fuzzy rules (CNN-FR) is a hybrid technique with powerful intelligent techniques in brain tumor classification and analysis. The aim of the combination of these two powerful methods is to improve the optimization rate and enhance the imaging quality of the tumor. The Neuro-fuzzy system was incorporate in imaging classification and examination since it contained knowledge representation. According to some researchers of neuro-fuzzy, it implements an uncertainty rule. Fuzzy logic focuses on knowledge representation and reasoning under uncertainty, we intend to incorporate the concepts of fuzzy logic into a deep learning framework (Tuan-Linh Nguyen et al., 2019).

In this system, the IF-THEN rule is used to construct human knowledge in brain classification. Figure 5 demonstrates the schematic of the system and the parameters of the fuzzy rule system. Layer 1 is the input layer, layer 2 is the fuzzification layer, layer 3 is the rule layer, layer 4 control layer 5 is the defuzzification. Figure 6, below show the complete view of an ANFIS model of integration of fuzzy rules and convolutional neural network, the process contained two input:X, Y and one output f. The network consists of five layers as described in Figure 6.

Inputs Layer: This is the first layer of the model, which is also called the adjustable inputs layer. The layer contained both normal and abnormal brain images. They are equal to linear tax function multiple with the input layer and it's each cell in the neuron is transmitted crisp input value directly 
Figure 4. Complex Neuro-Fuzzy Rules Inference System.

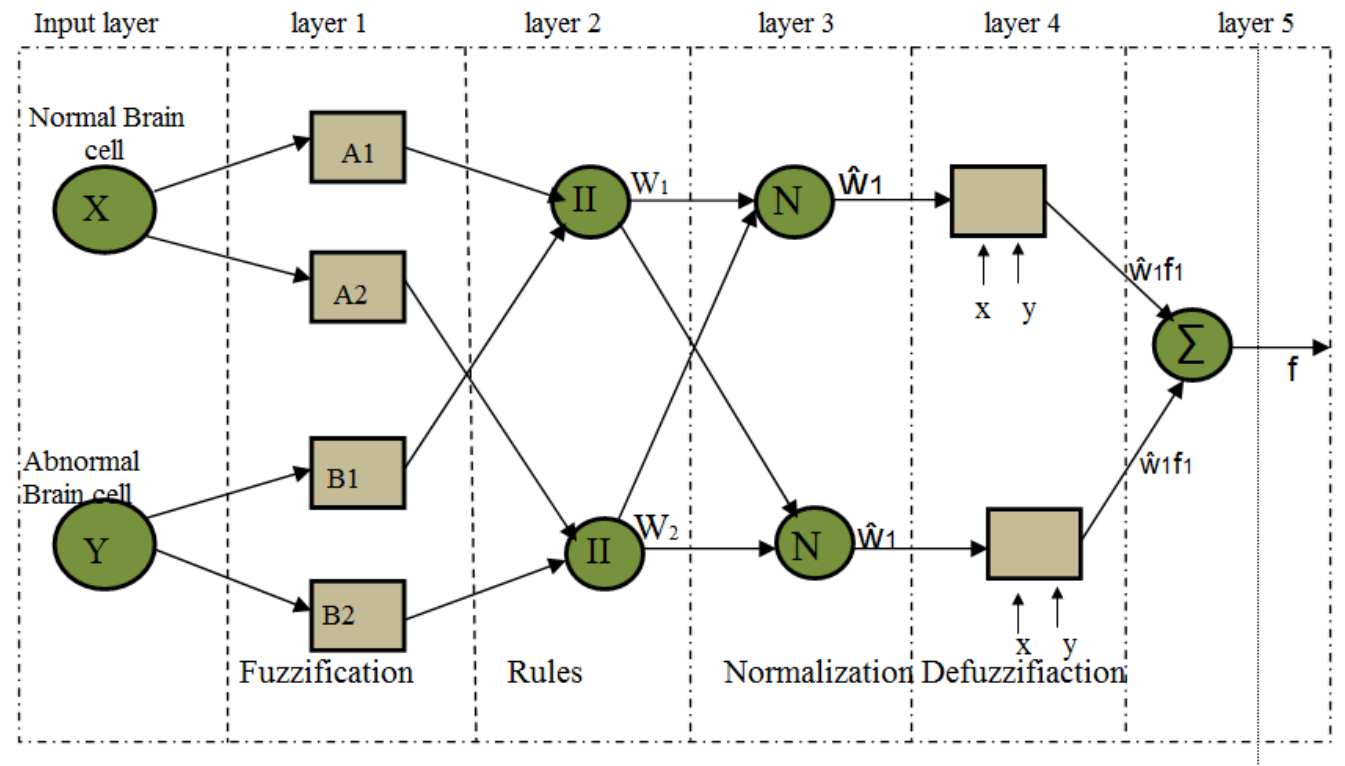

to the next layer which is represented as the input layers. That is $y \mathrm{i}=\mathrm{xi}$, which contained the normal brain and abnormal brain. Eqs 5 and 6 represent the new extraction features

$\mathrm{O} 1,_{\mathrm{i}}=\mathrm{UA}_{1}(\mathrm{x})$

$\mathrm{O} 1,_{\mathrm{i}}=\mathrm{uB}(\mathrm{y})$

where $\mathrm{x}$ the new features extraction as an input, $\mathrm{y}$ is the historical features dataset $(\mathrm{A}, \mathrm{B})$ is the linguistic variables, and $\{\mathrm{uAi}(\mathrm{x}), \mathrm{u} \mathrm{Bi}(\mathrm{y})$ are the membership functions.

First Layer: It is the real value of the linear tax function, which represents as, I1 and I2 values. The cells comprised four antecedents function that represented the fuzzy sets in the layers such as fellows: low (LW), high (H), small (SM), and large (LR). But each cell uses to determine, the kinetic values between one neuron layer to another the node in the cell which is represented as V. A fuzzification neuron receives a crisp input that determined the degree to which the input belongs in the fuzzy set. Besides the activation function of membership is set to specify the condition of brain cells in the fuzzy set. Based on the triangular sets of analysis of fuzzy rule theory, the activation functions are set to specify the parameters of the brain cells in layer 2.Crisp inputs are used to measure the sensitivity and pass the control to the system for further processing. The mathematical equations of the fuzzification layer base on the sum of kinetic values are given as follows:

$\mathrm{O} 2,_{\mathrm{i}}=\mathrm{W}_{\mathrm{i}}=\mu \mathrm{A}_{\mathrm{i}}(\mathrm{x}), \mathrm{i}=1,2$

$\mathrm{O} 2,_{\mathrm{i}}=\mathrm{W}_{\mathrm{i}}=\mu \mathrm{B}_{\mathrm{i}}(\mathrm{y}), \mathrm{i}=3,4$

where $\mathrm{i}=1,2$ and $\mathrm{W}_{1}$ is the output for each node and $\mathrm{O} 2$ is output of new neuron 
$\mathrm{x}$ and $\mathrm{y}$ are the inputs to node $\mathbf{I}, \mathbf{V i}$ are a linguistic label with low and High respectively. And their connection with this node function, $\mu \mathrm{Ai}(\mathrm{x}) \mu \mathrm{Bi}(\mathrm{x})$ and $\mu \mathrm{Ai}-2(\mathrm{y}) \mu \mathrm{Bi}-2(\mathrm{y})$, with the adoption of any fuzzy membership function. The equation below represents the membership function of brain tumor:

$$
\mu_{A i}(x)=\frac{1}{1+\left[\left(\frac{x-c_{i}}{a_{i}}\right)^{2}\right] b_{i}}, 1,2
$$

Differentiate the equation 9, above with exponentially function is given the equation 10 , as fellows

$$
\mu_{A i}(x)=\exp \left[-\left(\frac{x-c_{i}}{a_{i}}\right)^{2}\right]
$$

ai, bi, and ci are the parameters of the membership function. The theory is used for imaging medical classification and analysis based on the principle IF, AND, and THEN. But different rules share different output membership functions during their implementation.

Second Layer:. The cell layer corresponds to a single fuzzy rule. In this layer, we calculate the output (II) of layer three by multiple the U layer of the lower cell and the small cell ie ULW and USM. In the layers, we determine the fuzzy strength(w) of the products of (U) values

$\mathrm{O} 3, \mathrm{i}=\mathrm{W}_{1}=\mathrm{w}_{1} / \mathrm{w}_{1}+\mathrm{w}_{2}$

where $\mathrm{i}=1,2$

Fouth and Fifty Layers: Its layer represents a single output of the neuro-fuzzy system and the layer is fuzzy sets which converted crisp values and the strengths into a single set fuzzy.Besides the layers project the final output and control the system center of gravity(COG) in the tumor.In the defuzzification layer, we determined the normalized firing strength of each rule and product of normalized firing strength and output of the corresponding firing rule $y$.

$\mathrm{O} 4, \mathrm{i}=\mathrm{W}_{\mathrm{i}} \mathrm{f}_{1}=\mathrm{W}_{1}\left(\mathrm{p}_{1} \mathrm{x}+\mathrm{q}_{1} \mathrm{y}+\mathrm{r}_{1}\right)$

where consequent parameter $\left(\mathrm{p}_{1}, \mathrm{q}_{1}, \mathrm{r}_{1}\right), \mathrm{i}=1,2$

In the model, we integrate fuzzy rules which required the knowledge of reasoning and optimization to classified brain tumors based on the tumor brain cell or a normal brain cell. Hence, $\mathrm{T}$ is the desired output of the controlled plant and its value is always set to zero which means after controlling the object value of the error function would be a minimum value. Using the steepest gradient method for the error backpropagation algorithm.

Rule $_{(1)}:$ IF $x$ is $\mathrm{A}_{1}$ AND $y$ is $\mathrm{B}_{1}$, THEN

$f_{1}=p_{1} x+q_{1} y+r_{1}$

Rule $_{(2)}:$ IF $x$ is $\mathrm{A}_{2}$ AND $y$ is $\mathbf{B}_{2}$, THEN

$f_{2}=p_{2} x+q_{2} y+r_{2}$ 
$\mathrm{x}$ and $\mathrm{y}$ are the inputs,

$\mathrm{Ai}$ and $\mathrm{Bi}$ are the fuzzy sets,

fi is the outputs within the fuzzy region specified by the fuzzy rule, and, pi, qi, and ri are the design parameters that are determined during the training process. Each layer in the neuro-fuzzy system is associated with a particular step in the fuzzy inference process.

Layer $6=$ Output layer. In this layer, we summarized all the output calculated in the layer 4 and 5 and output them as on linear layer. The equation is given as follows:

$\mathrm{O} 5, \mathrm{i}=\mathrm{E}_{1} \mathrm{~W}_{1} \mathrm{f}_{1}=\mathrm{Ew}_{1} \mathrm{f}_{1} / \mathrm{Ew}_{1}, 1=1.2$

Output $=6 \mathrm{O} 1=\mathrm{W}_{1} \mathrm{y}_{1}+\mathrm{w}_{2} \mathrm{y}_{2}+\mathrm{W}_{4} \mathrm{y}_{4}+\mathrm{W}_{5} \mathrm{y}_{5}$

whereas the performance of the output depends on the membership function distribution of input variable: ai, bi, ci

$y_{1}^{(4)}=\sum_{j=1}^{n} y_{j}^{(3)} * \mathrm{w}_{j}$

$y_{2}^{(4)}=\sum_{j=1}^{n} y_{j}^{(3)}$

$y^{(5)}=\frac{y_{1}^{(4)}}{y_{2}^{(4)}}=\frac{\sum_{j=1}^{n} y_{j}^{(3)} * \mathrm{w}_{j}}{\sum_{j=1}^{n} y_{j}^{(3)}}$

Generally, to control the secondary source error which is not always equal strength and $1 / \mathrm{c} 0$ time delay as the primary source at the downstream of the duct. Hence the error exists between the output $y(5)$ of the fuzzy neural network and the real controlled plant. To reduce the error source in the classification and identification of the tumor, we defined an error function $\mathrm{E}$ :

$\mathrm{E}=1 / 2\left[\mathrm{y}^{(5)}-\mathrm{T}\right] 2$

Base, on the mathematical model of procedures above, we use the ANFIS approach(ANFIS Fuzzy Takagi Sugeno method(Rahouma, Kamel H., et al 2017). Using CNN integrated with fuzzy rules to classify the brain tumor features and evaluate the outcome of the future complication of the brain cells. The following algorithm slow the method of classification and evaluation method. 
Start

(1) Data inputs consist of two stages:

(a) Present feature for current images(Testing data)

(b) The data set of

(c) features of degrees of the tumor(Training data)

(2). For each image feature do the following:

Start a loop

(a) Apply the partitioning of the "FUZZY RULE" function with the data set of the images features

(b) Select the parameter of the partitioning function

(a) Choose the membership function of our design

(b) Select the type of input function

(c) Select the type of input function(linear)

(c)To train ANFIS structure, insert the important parameters for this structure

(a)Select type of optimization(Hybrid)

(b)Choose the number of epochs

(3). Compute the error

Err $=($ actual value-Estimated value $/($ actual value $) * 100 \% \% \%$ using for estimation of classification

End loop

(a)Calculate accuracy

(b)Compute the accuracy $=1$-Err

(c)Print the results(degree of the tumor and classification features

End

\section{EXPERIMENTAL RESULTS AND DISCUSSION}

In this section, we present the experimental results with a discussion. The experiment's implementation of the model has been carried out with the fellow's steps: a collection of the dataset, data visualization, data augmentation, data splitting, building the model, pre-training the model, actual training model, and evaluation model. The details of each step are explained in the next paragraph and complete information was provided base on the implementation.

\subsection{MRI Image}

MRI (Magnetic Resonance Imaging) is a scanner that uses powerful magnets to polarize excite hydrogen nuclei (a single proton) in human tissue, produces a single proton that can be detected and encoded spatially, in an image of the human body. Our experimental dataset was collected from the Website [http://www.diacom.com]. The dataset consists of a total of 3264 T1-CE MRI images with three different brain tumor types:100 brain tumor images of glioma tumor,115 brain tumors of meningioma tumor,74 brain tumors of pituitary tumor, and 105 brain cells without tumor. These images are divided into three categories $70 \%$ for training, $15 \%$ for testing, and $15 \%$ for validation. The implementation of the model is categorized as follows:(1) Training of the model, (2) Implement the model, (3) Evaluate the model. Therefore, the data visualization was implemented once after the dataset collected to know the component and labeled of the magnetic resonance image, it consists the fellows:100 brain tumor images of glioma tumor,115 brain tumors of meningioma tumor,74 brain tumors of pituitary tumor, and 105 brain cells without tumor, 3159 are labeled "Yes" which indicates that there are present of tumors and 105 are labeled "No" which indicates that the absence of tumors. The dataset was divided base on its categories but during the process of spitting, we have a problem of data imbalance in the region due to an insufficient lack of data variation. At this point, we apply data augmentation to regulate the dataset sorts out, image rotation, and flipping of the images. Figure 6, shows the features of our image dataset when training with a convolutional neural network combined with fuzzy.

A Tumor Brain Cell (b) A Non Tumor Brain Cell. 
Figure 5. MRI dataset
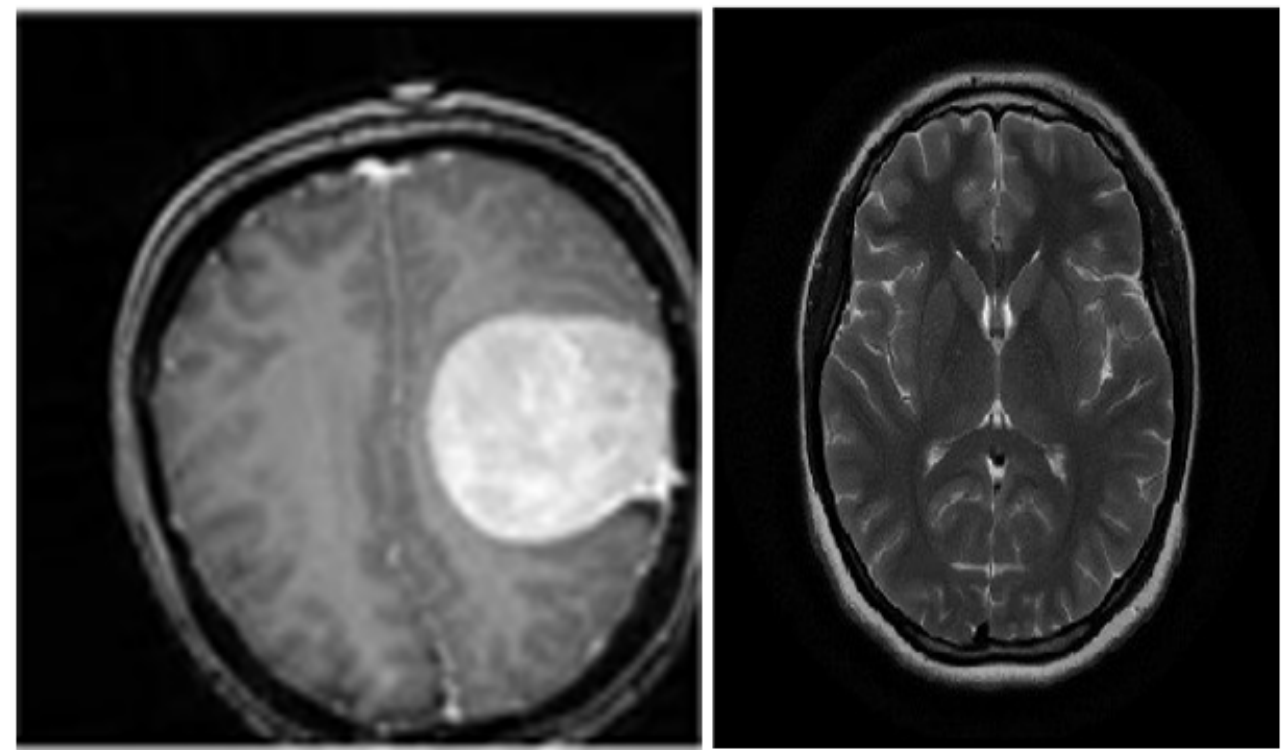

Figure 6. Image augmentation
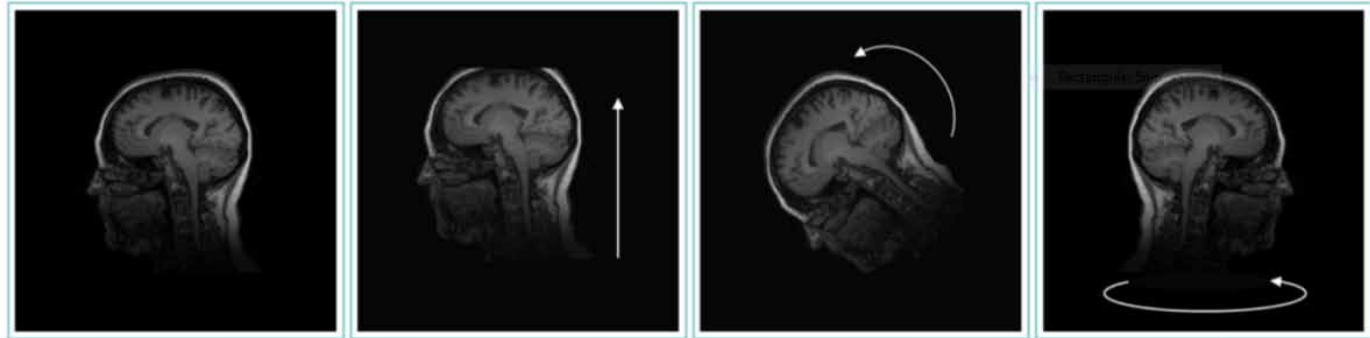

\subsubsection{Original (b) Translated (c) Rotated (d) Flipped}

During implementation, the dataset is divided into two parts(a) a training set using $80 \%$ of total images(2611.2 images), and testing set using $20 \%$ of the dataset. These percentages of implementation are referenced in the conditions specified in the dataset above. Moreover, full knowledge of CNNFL was applied in the classification of the tumors. We designed the model with CNN-FL build in google collab with convolutional, pooling layers, and fuzzy rules principle. As shown, in Figure 4 in the proposed model above which contained many sequences that help in memorizing image data image visualization and logic reasoning. The training of the model is divided into two such as(1) pretraining of a model(2), actual training. The pre-training of the model involves setting up the condition of fuzzy rules in the model such as IF, THEN, and AND in the convolutional neural network during the implementation. Once after pre-training of the model comes actual training of the model which we set the image dataset mode of the neural network environment and the images of the dataset are set in 1000 epochs. Each epoch can be thought of as an iteration in which we feed the training images again and again for the convolutional neural network to get trained better with the training images 
(Figure 7). In the evaluation of the model, we compared it with the real image images in the model. Then, in the experiment, we compared the performance of the model with the predictive models in terms of the following: accuracy, sensitivity, and specificity as shown in Equation 21, 22, and 23.

Accuracy. Described the degree of closeness of estimating the original value of the brain tumor during the classification process. It is the proportion of the total number of predictions. The accuracy is defined in $\mathrm{Eq}(21)$ as fellows

$$
\text { Accuracy }=\frac{T P+T N}{T N+F P+F N+T P}
$$

where Tp, Tn, Fp, and Fn represent true positive, true negative, false positive, and false negative, respectively.

Sensitivity.Described the ratio of positives value for identifying the tumor classification using CNN- FL. It is the rate of measure the classifier performance. It is mathematically represented.

Sensitivity $=\frac{T P}{T P+F N}$

where $\mathrm{TP}=$ true positive and $\mathrm{FN}=$ false negative.

Specificity. Described the ratio of positives value for identifying the tumor classification using CNN- FL. It is the rate of measure the classifier performance. It is mathematically represented.

Specificity $=\frac{T N}{T N+F P}$

where $\mathrm{TN}=$ true negative and $\mathrm{FP}=$ false positive

Figure 7. Sample of the images outcome
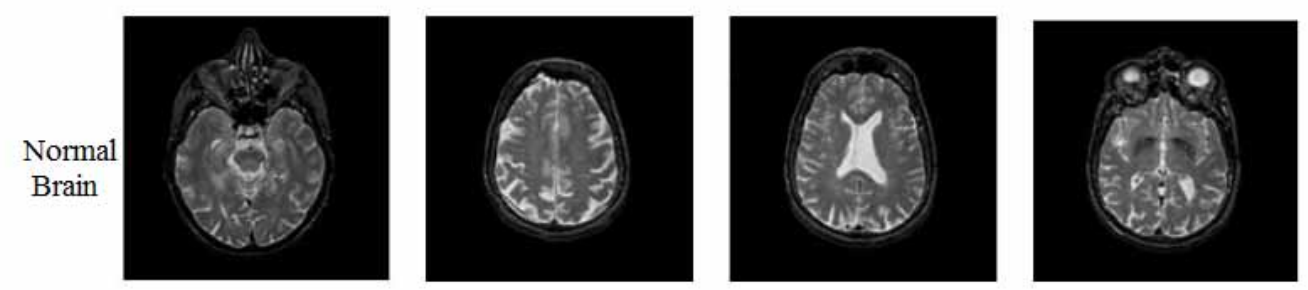

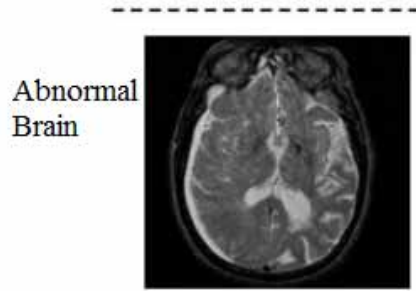

Ependymoma

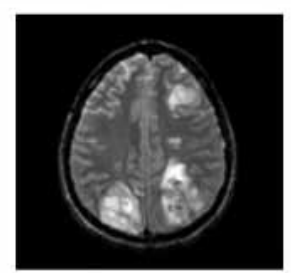

Pilocytic astrocytoma.

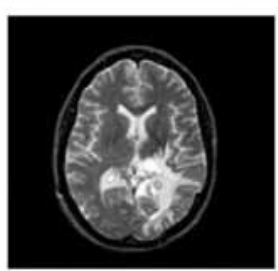

Sarcoma

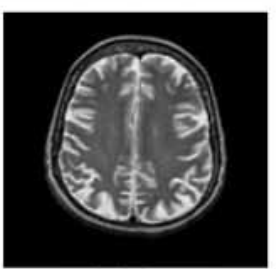

Glioma 


\subsection{Experimental Results}

In our experimental process, the proposed model has been implemented by dividing the dataset into two different parts:(a) training set $80 \%$, and (b) testing $20 \%$. These percentages are as referenced in the conditions specified in the dataset [http://www.diacom.com]. The experimental results are set out in Table 1. The experiments employed common standard performance measures: specificity, sensitivity, accuracy, and F-measure to evaluate the outcome performance of the comparative models. A comparative analysis compared the performance of the current proposed model deep convolutional neural networks with transfer learning for automated brain image classification and classification of tumors in human brain MRI using wavelet and support vector machine methods. Initially, we implemented a piloting stage to validate the proposed model both manually and automatically to supervise the optimization techniques of (CNN-FL) based on medical imaging classification and detection. Then, we continued the experimental testing using the input data to determine if the changes made in the original model improved the accuracy of the classification in the brain cells.

Table 2. Evaluation and Comparison experimental results of brain tumor classification

\begin{tabular}{|l|l|l|l|l|l|l|l|l|}
\hline \multicolumn{1}{|c|}{ Techniques } & $\begin{array}{c}\text { Total } \\
\text { Images }\end{array}$ & TP & FN & TN & FP & $\begin{array}{c}\text { Specificity } \\
\%\end{array}$ & $\begin{array}{c}\text { Sensitivity } \\
\%\end{array}$ & $\begin{array}{c}\text { Accuracy } \\
\%\end{array}$ \\
\hline $\begin{array}{l}\text { Classification of Tumors in Human Brain } \\
\text { MRI using Wavelet and Support Vector } \\
\text { Machine [14] }\end{array}$ & 75 & 64 & 1 & 7 & 3 & 70 & $98.46 \%$ & $94.7 \%$ \\
\hline $\begin{array}{l}\text { Deep convolutional neural networks with } \\
\text { transfer learning for automated brain image } \\
\text { classification [15] }\end{array}$ & 100 & 100 & 3 & 8 & 3 & 100 & 87.5 & $95.0 \%$ \\
\hline $\begin{array}{l}\text { Convolutional Neural Network integrated with } \\
\text { fuzzy Logic (CNN- FL) [proposed model] }\end{array}$ & 1163 & 700 & 300 & 400 & 300 & 700 & $95.5 \%$ & $97.6 \%$ \\
\hline
\end{tabular}

Experimental results in Table 2 show the evaluation of brain tumor classification and comparison of different proposed models. Moreover, our experimental results obtained was evaluated using the standard metrics and parameters our proposed model achieved an accuracy of $97.6 \%, 95.0 \%$, and $94.7 \%$ for the proposed model, Deep convolutional neural networks with transfer learning for automated brain image classification and Classification of Tumors in Human Brain MRI using Wavelet and Support Vector Machine respectively. The proposed CNN-FL model, in achieving an accuracy of $97.6 \%$ demonstrated a strong impact on the results in the experimental testing. It demonstrated that the current model is optimization the time and improved medical imaging.

\subsection{Comparison Criteria}

Our experimental results were compared based on the challenges we face during the implementation such as fellows, dataset, accuracy, and techniques used in the proposed model and other alternative others. Table 1, shown complete full results obtained from the proposed model analysis and alternative comparison model base on the three critical parameters such as dataset, accuracy, and techniques. Tables 2, 3 and figures showed the output of the criticism. Because of short-term load data, and to show how the proposed model was capable to solve complex classification problems, we have selected them to validate and the proposed model. The results of the comparison proved that convolutional neural network combined with fuzzy rule achieves good results in the medical imaging classification

(A). Comparative Analysis of the Proposed Model Dataset and Other Alternative Models 
Data load is the most complicated issue we face in the implementation because the data is considered as nonlinear and Intra seasonality which makes solving this problem even harder (http:// www.diacom.com). Because of the difficulty that exists in using short-term load data, and to show how the proposed method was capable to solve a complex classification problem. As applied longterm load data and validate the proposed model the issues since the resolved.

\section{Evaluation and Comparison of the Proposed Model Base on Data Sets}

Figure 8. model comparison

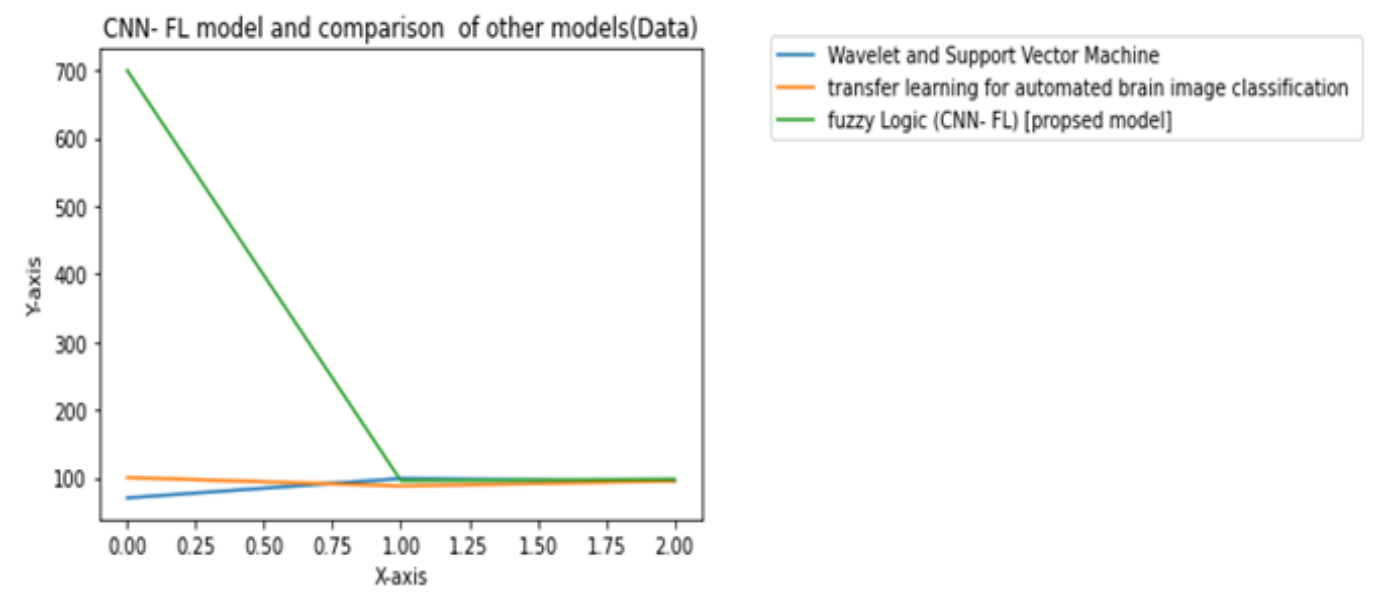

Figure 8 shows the results obtained in the comparative analysis of the three different proposed models considered. The graph explained the features of the different models base on the dataset. From Figure 8 we can see that in computation using the standard performance measures our proposed model demonstrates high performance in comparison to other approaches considered. Moreover, our proposed model demonstrated a high degree of accuracy in the classification and optimization of brain tumors. The rising of the current model in the graph claim that our proposed model may be used by domain experts to enable effective medical imaging classification.

\subsubsection{Comparative Analysis of Proposed Model Accuracy, Optimization, and Other Alternative Models}

The comparative analysis of the proposed model of CNN with Fuzzy rules in the identification and classification of brain tumors based on the normal and abnormal brain cells. This current proposed model was compared with other existing methods such as Classification of Tumors in Human Brain MRI using Wavelet and Support Vector Machine [14] and Deep convolutional neural networks with transfer learning for automated brain image classification[15], of specificity, sensitivity, and accuracy using Web sites database for verifying training data percentages is shown in Table 1.The specificity values measured by Classification of Tumors in Human Brain MRI using Wavelet and Support Vector Machine $\operatorname{model}(($ Specificity $=70 \%$, sensitivity $=98.46 \%$ and accuracy $=94.7 \%)$ and Deep convolutional neural networks with transfer learning for automated brain image classification model(Specificity $=100 \%$, sensitivity $=87.5 \%$ and accuracy $=95.0 \%$ ). . The proposed Dolphin SCA-based Deep CNN shows the highest accuracy among the existing methods and thus, the ability to identify the tumorous. Table 3 displays the comparative analysis plot based on the maximum iteration and CUP time using 
to evaluate the level of the analysis for the tumors. The analysis is based on the number of iteration and values ranging from 100-1000 and 500- 1000 with times varying system.

Table 3. Comparison of the rate of optimization of CNN-FL model and CNN model

\begin{tabular}{|c|c|c|c|}
\hline Method for feature extraction & Maximum iteration & CPU time (minute) & Accuracy of (CNN-FL) \\
\hline CNN & $100-1000$ & $2 \min$ & $95.0 \%$ \\
\hline CNN- FL & $500-1000$ & $3 \min$ & $97.6 \%$ \\
\hline
\end{tabular}

Table 3 shows the comparison of the optimization rate of the iteration of the CNN model and CNN-FL model during the experiment with the time rate of CPU. In the analysis of the accuracy, the CNN-FL model gives an accuracy of $97.6 \%$ while the CNN model gives an accuracy of $95.0 \%$. In conclusion, the current proposed model gives $97.6 \%$ with an error rate of $2.4 \%$. Figure 9 shows

Figure 9. comparison of optimization rate of accuracy

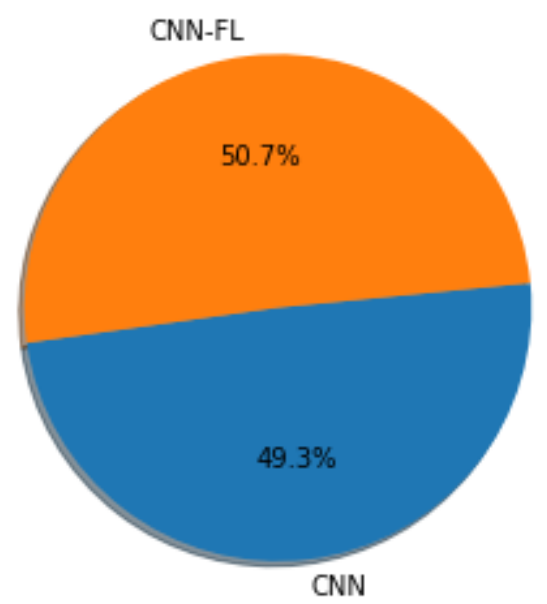

the analysis and comparison of optimization rate of the iteration accuracy of the CNN-FL model and CNN model

An analysis of the optimization rate of the iteration during the experiments. The pie chart diagram showed the percentage of the CNN-FL and CNN models with an accuracy of $50.7 \%$ and $49.3 \%$ as shown in Figure 9. The iteration values represent the rate of optimization of the algorithm during the diagnosis.

Table 4 and Figure 10, shows a comparison of various techniques with their performance accuracy model, CNN-FL $97.6 \%$ with an error rate of $2.4 \%$ on best-selected features. In comparison with other publication on brain tumors in 2020, CNN and NADE 95.0\%, OGSE, and PGSE 97.0\%, CNN and Resnet50 97.2\%, CNN and transfer learning 90.0\%, Dolphin-SCA and CNN 96.3\%, CNN and SR-FCM 98.3\%, CNN and GAN 95.6\% CNN and FL 97.6\%. For a fair comparison of the other techniques on brain tumors, $\mathrm{CNN}$ has a variety of data methods and automated approach in the classification. On the other hand, the current proposed model CNN-FL obtained accuracy is $97.6 \%$ 
Table 4. Evaluation of different techniques and alternative related model

\begin{tabular}{|l|l|l|l|l|}
\hline \multicolumn{1}{|c|}{ S/N } & \multicolumn{1}{c|}{ Authors } & \multicolumn{1}{c|}{ Years } & \multicolumn{1}{c|}{ Method } & \multicolumn{1}{c|}{ Accuracy } \\
\hline 1 & Raheleh Hashemzehi a et al & 2020 & CNN and NADE & $95.0 \%$ \\
\hline 2 & T. Maekawa et al & 2020 & OGSE and PGSE & $97.0 \%$ \\
\hline 3 & Ahmet Çinar et al & 2020 & CNN and Resnet50 & $97.2 \%$ \\
\hline 4 & Priyansh Saxena et al & 2020 & CNN, and transfer learning & $90.0 \%$ \\
\hline 5 & Sharan Kumar et al & 2020 & Dolphin-SCA and Deep CNN & $96.3 \%$ \\
\hline 6 & Fatih Özyurt et al & 2020 & CNN and SR-FCM & $98.3 \%$ \\
\hline 7 & Navid Ghassemi Fong et al & 2020 & CNN and GAN & $95.6 \%$ \\
\hline 8 & Proposed model & 2020 & CNN and FL & $97.6 \%$ \\
\hline
\end{tabular}

Figure 10. Analysis of different techniques use for brain tumor classification

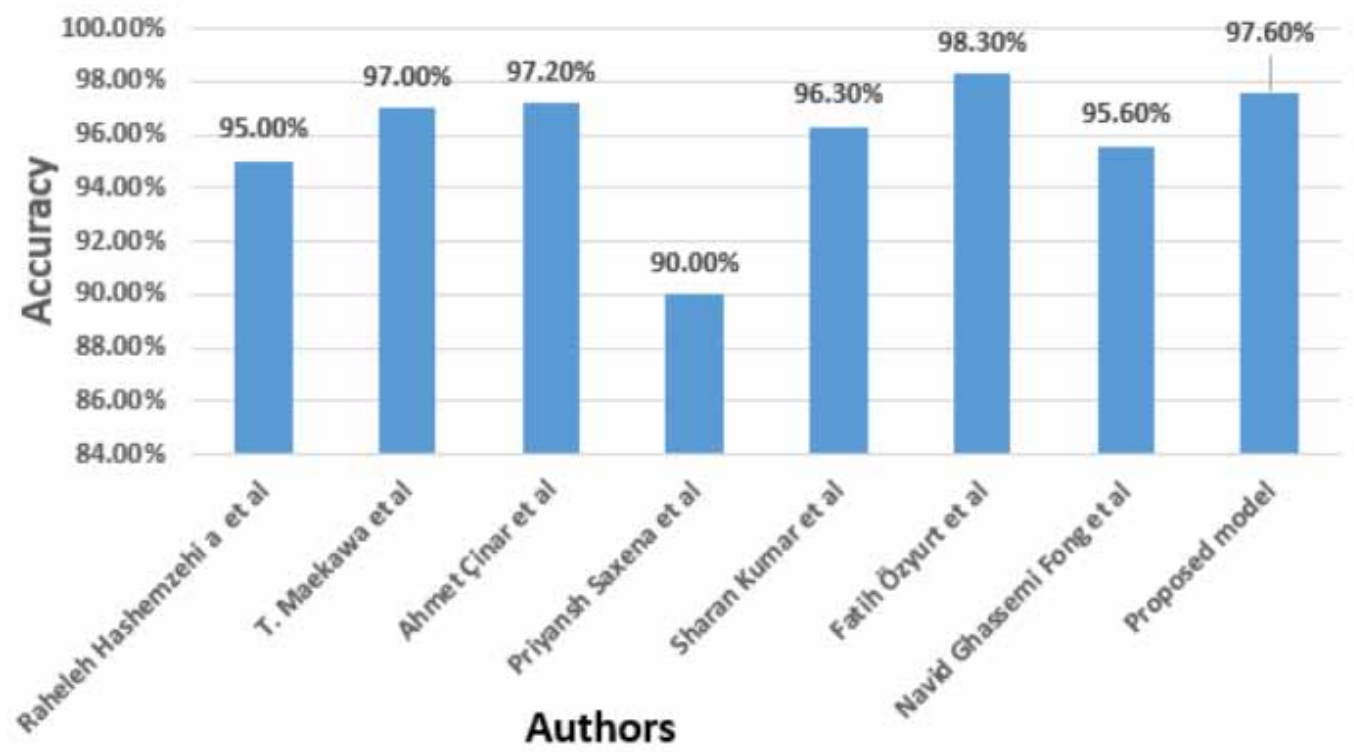

which is the second-best technique. Moreover, Figure 8 also summarized the features of techniques uses in the comparison through the histogram method.

\section{CONCLUSION AND FUTURE WORK}

This work has addressed the potential approach, to distinguished and classified the brain cell base on the tumor brain cell or non-tumor brain cell and pointed out the solution to improve the problems medical experts during the analysis of tumor brain cells. The proposed model work in the following: (1) To classify brain cells toward abnormal brains and normal brains. (2) To enhance the Neuro-Fuzzy rules in areas of medical imaging classification. The experimental results of the proposed model prove 
that the proposed approach as currently constituted achieves improved identification and classification performance as compared to the alternative approaches evaluated in our comparative analysis. Based on the research work show that the proposed model has the potential to assist in identification and classification to arrive faster, optimization, and more accurate in classification. Moreover, in response to future work, we proposed a model of segmentation of brain tumors using a convolutional neural network $(\mathrm{CNN})$ integrated with Generative Adversarial Network (GAN).

\section{ACKNOWLEDGMENT}

This research is funded by Vietnam National Foundation for Science and Technology Development (NAFOSTED) under grant number 102.05-2019.316. 


\section{REFERENCES}

Abd-Ellah, Awad, Khalaf, \& Hamed. (2016). Design and implementation of a computer-aided diagnosis system for brain tumor classification. International Conference on Microelectronics (ICM), 28(3), 73-76. doi:10.1109/ ICM.2016.7847911

Badran, Mahmoud, \& Hamdy. (2010). An algorithm for detecting brain tumors in MRI images. International Conference on Computer Engineering \& Systems, 5(2), 368-373. doi:10.1109/ICCES.2010.5674887

Badran, E. F., Mahmoud, E. G., \& Hamdy, N. (2010). An algorithm for detecting brain tumors in MRI images. International Conference on Computer Engineering \& Systems, 5(2), 368-373. doi:10.1109/ICCES.2010.5674887

Bhanumurthy \& Anne. (2014). Automated detection and segmentation of tumor in brain MRI using artificial intelligence. IEEE International Conference on Computational Intelligence and Computing Research Coimbatore, 6(10), 1-6. doi:10.1109/ICCIC.2014.7238374

Çinar, A., \& Yıldırım, M. (2020). Detection of tumors on brain MRI images using the hybrid convolutional neural network architecture. Medical Hypotheses, 139(6), 139-109684. PMID:32240877

Collobert, R., \& Weston, J. (2008). A Unified Architecture for Natural Language Processing: Deep Neural Networks with Multitask Learning. Proceedings of the 25th international conference on Machine learning, 25(8), 168-175. doi:10.1145/1390156.1390177

Domenico, Ch., Pasquale, Ch., \& Domenico, L. (2020). An update of the imaging and diagnostic techniques in use for the preservation of eloquent areas in brain tumor surgery-An opinion paper Interdisciplinary Neurosurgery. Interdisciplinary Neurosurgery: Advanced Techniques and Case Management, 20(5), 100-611.

Fatih, Ö., Eser, S., \& Derya, A. (2020). An expert system for brain tumor detection: Fuzzy C-means with superresolution and convolutional neural network with extreme learning machine. Medical Hypotheses, 134(1), 134-109433. PMID:31634769

Ghassemi, N., Shoeibi, A., \& Rouhani, M. (2020). A deep neural network with generative adversarial networks pre-training for brain tumor classification based on MR images. Biomedical Signal Processing and Control, 57(3), 101678-101688. doi:10.1016/j.bspc.2019.101678

Hashemzehi, R., Mahdavi, S. J. S., Kheirabadi, M., \& Kamel, S. R. (2020). Detection of brain tumors from MRI images based on deep learning using. Biocybernetics and Biomedical Engineering, 40(3), $1225-1232$. doi:10.1016/j.bbe.2020.06.001

Hiroyuki, S., \& Masashi, K. (2019). Automatic Detection of a Standard Line for Brain Magnetic Resonance Imaging Using Deep Learning. Brain detection. Applied Sciences (Basel, Switzerland), 9(18), 18-3849.

Huang, Z., Xu, H., Su, S., Wang, T., \& Luo, Y., Zhao, X., \& Zhao, Y. (2020). A computer-aided diagnosis system for brain magnetic resonance imaging images. Computers in Biology and Medicine, 6(121), 1-103818. PMID:32568685

Kaur, T., \& Gandhi, T.K. (2020). Deep convolutional neural networks with transfer learning for automated brain image classification. Machine Vision and Applications, 31(3).

Khan, M. A., Rashid, M., Sharif, M., Javed, K., \& Akram, T. (2019). Classification of gastrointestinal diseases of the stomach from WCE using improved saliency-based method and discriminant features selection. Multimedia Tools and Applications, 78(7), 27743-27770. doi:10.1007/s11042-019-07875-9

Krizhevsky, A., Sutskever, I., \& Hinton, G. E. (2017). Imagenet classification with deep convolutional neural networks. MageNet classification with a deep convolutional neural network. Advances in Neural Information Processing Systems, 60(6), 105-1097.

Lakshmanaprabu, S., Sachi, K., Nandan, M., Shankar, K., Arunkumar, N., \& Gustavo, R. (2019). The optimal deep learning model for classification. Future Generation Computer Systems. Future Generation Computer Systems, 92(3), 374-382.

Maekawa, Hori, \& Murata. (2020). Differentiation of high-grade and low-grade intra-axial brain tumors by time-dependent diffusion MRI. Magnetic Resonance Imaging, 72(10), 34-41. 
Michael, I., Byung, C., Yoon, J., Heit, M. D., Nancy, F., \& Max, W. (2018). Current Clinical State of Advanced Magnetic Resonance Imaging for Brain Tumor Diagnosis and Follow Up. Seminars in Roentgenology, 53(1), 45-61. doi:10.1053/j.ro.2017.11.005 PMID:29405955

Mohamed Shakeel, P., Tobely, T. E. E., Al-Feel, H., Manogaran, G., \& Baskar, S. (2019). Neural Network Based Brain Tumor Detection Using Wireless Infrared Imaging Senso. IEEE Access: Practical Innovations, Open Solutions, 10(7), 5577-5588. doi:10.1109/ACCESS.2018.2883957

Mohan, G., \& Subashini, M. M. (2018). MRI based medical image analysis: Survey on brain tumor grade classification. Biomedical Signal Processing and Control. Biomedical Signal Processing and Control, 39(2), 139-161. doi:10.1016/j.bspc.2017.07.007

Mohan, G., \& Subashini, M. M. (2018). MRI based medical image analysis: Survey on brain tumor grade classification. Biomedical Signal Processing and Control, 39(1), 139-161. doi:10.1016/j.bspc.2017.07.007

Moore, P., \& Pham, H. V. (2012). Intelligent context with decision support under uncertainty. Sixth International Conference on Complex, Intelligent, and Software. doi:10.1109/CISIS.2012.17

Mubashir, A., Mahmood, H., Imran, Sh., \& Abdelrahman, O. (2012). Classification of Tumors in Human Brain MRI using Wavelet and Support Vector Machine. IOSR Journal of Computer Engineering, 8(2), 25-31. doi:10.9790/0661-0822531

Bishop. (2016). Pattern Recognition and Machine Learning, Pattern recognition. Information Science and Statistics. Journal of Electronic Imaging, 16(4), 140-155.

Priyansh, S., Akshat, M., Shivani, T., \& Saumil, M. (2019). Predictive modeling of brain tumor: A Deep learning approach. In Innovations in Computational Intelligence and Computer Vision (ICICV-2020). Springer.

Rahouma, K. H. (2017). Analysis of Electrocardiogram for Heart Performance Diagnosis Based on Wavelet Transform and Prediction of Future Complications Machine learning and prediction Techniques for Biomedical signal. Prediction Behavior Technique, 41(4).

Roy, S., Sadhu, S., Bandyopadhyay, S. K., Bhattacharyya, D., \& Kim, T. H. (2016). Brain tumor classification using adaptive neuro-fuzzy inference system from MRI. International Journal of Bio-Science and Bio-Technology, 8(3), 203-218. doi:10.14257/ijbsbt.2016.8.3.21

Saroj, K., Chandra, M., \& Kumar, B. (2020). Fractional mesh-free linear diffusion method for image enhancement and segmentation for automatic tumor classification. Biomedical Signal Processing and Control, 58(2), 1-101841.

Sharan, K., Dattatreya, P., \& Mankame, B. (2020). Optimization drove Deep Convolution Neural Network for brain tumor classification. Biocybernetics and Biomedical Engineering, 40(3), 1190-1204. doi:10.1016/j. bbe.2020.05.009

Srivastava, N., Hinton, G., Krizhevsky, A., Sutskever, I., \& Salakhutdinov, R. (2014). Dropout: A simple way to prevent neural networks from overfitting. Journal of Machine Learning Research, 15(2), 1929-1958.

Thomas, A. M., Moore, P., \& Xhafa, F. (2014). Smart care spaces: Pervasive sensing technologies for at-home care. International Journal of Ad Hoc and Ubiquitous Computing, 16(4), 268-282. doi:10.1504/IJAHUC.2014.064862

Tuan, L. N., Swathi, K., \& Minho, L. (2019). A multimodal convolutional neuro-fuzzy network for an emotional understanding of movie clips. Neural Networks, 118(10), 208-219. PMID:31299625

Tuan, T. M., Duc, N. T., \& Pham, V. H. (2017). Dental diagnosis from X-ray images using fuzzy rule-based systems. International Journal of Fuzzy System Applications, 6(1), 1-16. doi:10.4018/IJFSA.2017010101

Xiaoheng, J., Yanwei, P. J. P., \& Yinghong, X. (2018). Deep neural networks with Elastic Rectified Linear Units for object recognition. Neurocomputing, 276(31), 1132-1139.

Yang, A., Yang, X., Wu, W., Liu, H., \& Zhuansun, Y. (2019). Research on Feature Extraction of Tumor Image Based on Convolutional Neural Network. IEEE Access: Practical Innovations, Open Solutions, 19(1), 24204 24213. doi:10.1109/ACCESS.2019.2897131 
Eloanyi Samson Amaechi is a researcher and Ph.D. student at Hanoi University of Science and Technology (HUST), Hanoi, Vietnam in the Department of Information Systems. His research work focuses on applying deep learning Networks in the global scientist world such as optimization, decision-making system, and big data analysis.

Hai Van Pham has received a Doctorate of Engineering degree (Ph.D.) at Ritsumeikan University (Japan). He is an Associate Professor at School of Information and Communication Technology, Hanoi University of Science and Technology. His major fields include Artificial Intelligence, Knowledge Based, Big data, Soft Computing, Rulebased Systems and Fuzzy Systems. Dr. Hai Van Pham is an Associate Editor of International Journal of Computer Applications in Technology and other domestic and international journals. He also serves as Chairs and Co-chairs of organized several sessions at international conferences such as KSE 2019, KSE 2017, KSE 2015, SOICT 2014. 